\title{
Unsupervised group feature selection for media classification
}

\author{
Maia Zaharieva $^{1}\left(\right.$ C $\cdot$ Christian Breiteneder $^{1} \cdot$ Marcus Hudec $^{2}$
}

Received: 16 March 2017 / Revised: 2 May 2017 / Accepted: 8 May 2017 / Published online: 25 May 2017

(C) The Author(s) 2017. This article is an open access publication

\begin{abstract}
The selection of an appropriate feature set is crucial for the efficient analysis of any media collection. In general, feature selection strongly depends on the data and commonly requires expert knowledge and previous experiments in related application scenarios. Current unsupervised feature selection methods usually ignore existing relationships among components of multi-dimensional features (group features) and operate on single feature components. In most applications, features carry little semantics. Thus, it is less relevant if a feature set consists of complete features or a selection of single feature components. However, in some domains, such as content-based audio retrieval, features are designed in a way that they, as a whole, have considerable semantic meaning. The disruption of a group feature in such application scenarios impedes the interpretability of the results. In this paper, we propose an unsupervised group feature selection algorithm based on canonical correlation analysis (CCA). Experiments with different audio and video classification scenarios demonstrate the outstanding performance of the proposed approach and its robustness across different datasets.
\end{abstract}

Keywords Feature selection - CCA - Audio classification Video classification

Maia Zaharieva

maia.zaharieva@tuwien.ac.at

Christian Breiteneder

christian.breiteneder@tuwien.ac.at

Marcus Hudec

marcus.hudec@univie.ac.at

1 TU Wien (TUW), Interactive Media Systems, Vienna, Austria

2 University of Vienna, Data Analytics and Computing, Vienna, Austria

\section{Introduction}

The selection of an appropriate feature set is a crucial step in the development of any approach for content-based media classification. The decision commonly depends on the specific characteristics of the underlying data, the target application scenario, and the application domain. In the context of media classification, usually a large number of features are employed to cope with the simple and usually rather syntactic nature of individual features. This is based on the assumption that different features describe different and complementary (orthogonal) qualities of the underlying data. Such feature sets commonly include features that are dependent on each other and features that are not at all relevant, which induces unnecessary computational costs and may lead to overfitting. Additionally, the high dimension may cause further problems, usually referred to as the curse of dimensionality.

Numerous methods have been developed to reduce the dimension and at the same time to reveal the same degree of class discrimination. Such methods can be grouped into dimension reduction and feature subset selection approaches. The most widely used linear dimension reduction method is principal component analysis (PCA), which in general transforms a set of highly correlated features into a smaller set of linearly uncorrelated variables called principal components. Usually, these components cannot be directly or easily interpreted. Feature selection tries to identify the most important and, additionally, less redundant features from a set of potential features. Existing approaches fall into four main categories based on the evaluation criterion applied: embedded, wrapper, filter, and hybrid methods [28,54]. In our approach, we employ a filter-based method, which means that the properties of the data are used as a criterion for the 
feature selection and the selected features can be passed on to any classifier.

In this paper, we introduce a group feature selection approach based on canonical correlation analysis (CCA). In general, CCA coefficients indicate the correlation between two features of varying dimensionality [20]. Large coefficients signify high correlations and, therefore, such coefficients can be indicators for redundant features. On the opposite end of the scale, low-correlated features provide additional information which can improve the descriptive power of the feature set. In previous work, we explored the correlation-based ranking of feature pairs in order to perform feature selection $[45,46]$. In contrast to our previous approach, in this work we investigate each feature separately with respect to the currently selected feature set, improving both the quality and the classification performance of the selected features.

In general, differences in the objectives of a feature selection process can be found in related work [7]. Improving the performance (efficiency) and providing faster and more costeffective (effectiveness) predictors are commonly addressed goals. Similar to some other authors [15], we add a third goal that we consider central to our approach: a better understanding of the underlying data. Most approaches for feature selection and dimensionality reduction do not differentiate whether or not a feature consists of several components. These methods usually do not aim at selecting entire features, but aim at the selection of components only. Features selected are then (linear) combinations of input features which makes the interpretation of data more complex. In the presence of multi-dimensional features that carry significant semantics, we therefore require input feature components to be kept together. We use the term group feature to emphasize this fact. The consideration of group features offers various advantages: First and most important, features consisting of several components and especially, when carrying significant meaning, should not be torn apart. Second, group features are less at risk of overfitting and show less sensitivity to different datasets and application scenarios. Third, the approach is more cost-effective, since group features are usually computed at once.

Most of the existing feature selection approaches aim at the optimization for a specific application scenario. In such a context, feature selection is usually based on previous experiments in related application scenarios. The major drawback of such a strategy is the use of prior information about the content, such as the existence of different content elements for audio-based classification (e.g., speech, music) that may lead to a bias in the selection process. It seems to be general knowledge that in some cases even a slight alteration of the application scenario, such as the consideration of an additional genre for a genre recognition approach, may lead to questioning the appropriateness of the previously employed features. Potentially, this results in the (partly resource-demanding) calculation of features that may not even contribute to the classification performance of the underlying approach.

Therefore, we add as a fourth and last objective for our feature selection approach robustness (stability). Robustness measures the number of commonly selected features in different runs or experiments. For environments with slightly changing scenarios or frequently changing data, it is of eminent importance that features are robust enough to cope with these changes by still achieving good retrieval performances.

This paper includes the following contributions:

1. We propose an improved version of the CCA-based feature selection method presented in $[45,46]$ that essentially compares CCA values between a single feature candidate and the set of already selected features. The approach is unsupervised and maintains the interpretation of entire features as the underlying feature components are kept together.

2. We introduce robustness as a goal for feature selection in the case of changing media repositories and define a measure for robustness. We argue and demonstrate that many of the excellent results in media feature selection are clearly tailored to specific datasets and results drop immediately when the methods are confronted with larger variations in the data. The measure for robustness allows to compare different approaches in this respect.

3. We perform a thorough evaluation of the proposed approach on datasets from the audio and video domains with different application scenarios. Evaluation results demonstrate the outstanding performance of the approach and its robustness across different datasets. Our unsupervised approach is competitive to state of the art approaches that stress the importance of the "right" feature set and that, therefore, require careful manual selection of their features.

4. We question the appropriateness of application-oriented approaches at least for many tasks in the audio domain, specifically the ones investigated here, and favor a robust data-oriented approach for feature selection. We understand that the quality and robustness of features selected by our approach is primarily caused by the powerful features developed in the audio domain so far. We are aware that more evaluations for different datasets and different application scenarios will be necessary to investigate this issue further, but we believe that the method suggested will have an impact on how features will be selected in the audio domain in the future.

This paper is organized as follows. Section 2 presents an overview of related work. Section 3 presents proposed CCA-based feature selection approach. Section 4 outlines 
the evaluation setup for the performed experiments. Section 5 presents the results of an experimental study of the proposed approach and a comparison with representative methods. Section 6 concludes this work.

\section{Related work}

Feature selection is commonly applied to reduce the dimensionality of exploited data, to remove irrelevant items, to increase accuracy, and to improve result comprehensibility [15]. Feature selection approaches can be broadly divided into embedded, wrapper, filter, and hybrid methods $[28,54]$. Out of these four categories, filter approaches are widely employed for their efficiency and ability to generalize since they are not bound to the bias of any learning algorithm. Filter methods follow mainly two approaches for feature selection: individual feature evaluation and feature subset evaluation. Individual feature evaluation methods assess each feature component individually and assign weights according to their relevance, resulting in a ranked list of features $[17,29]$. Subset evaluation approaches assess features in the context of generated feature subsets [34,61]. Such methods iteratively generate feature subsets using different search strategies until a predefined stopping criterion is met. The evaluation of generated feature subsets usually employs some statistical measures to assess their relevance and redundancy. In this context, correlation analysis is often applied to measure feature redundancy. In our previous work, we proposed a CCA-based approach, that involves information gain as a feature selection rule [46]. We performed a thorough comparison with well-established filter-based selection methods, Chi-square [33], information gain [17], information gain ratio [42], ReliefF [29], consistency-based [34], and correlation-based [16]. Our approach outperformed the investigated algorithms in terms of accuracy and runtime and obtained a high robustness of the feature selections for different datasets in the same audio retrieval task.

Both subset and individual feature evaluation approaches ignore any existing relationships among the components of multi-dimensional features. Recently, Xu et al. [57] proposed a gradient boosted feature selection approach that is able to consider predefined group feature structures. However, the proposed approach employs the group structure information for controlled boosting only and, thus, components from the same multi-dimensional feature are, in general, preferred for selection. As a result, the final feature set still consists of single feature components (from potentially few multi-dimensional features). In contrast, the group lasso algorithm [62] is an extension of the popular lasso approach [55] for the selection of predefined groups of variables in regression models [35]. Although the estimates are invariant under (groupwise) orthogonal transformations [62], fitted models may not be sparse. Simon et al. propose sparse group lasso that yields solutions that are sparse at both group and individual feature levels $[13,52]$. However, Hall and Miller show that such model-based approaches may still be inadequate for detecting all influential features [18].

Current research on audio and video classifications focuses mostly on the development of new features and classification methods $[11,26,27,30,38]$. Few works exploit feature selection in the context of genre classifications $[9,12,22$, $50,60]$, musical instrument classification [8,51], and emotion/mood classification $[43,59]$, commonly using a single dataset only. Most approaches consider wrapper-based feature selection, i.e., the underlying feature selection process is supervised, maximizing the classification accuracy of a learning algorithm. Common approaches for the identification of potential feature sets include genetic algorithm [50] and greedy selection heuristics $[12,43,59,60]$. Although, usually, wrapper-based feature selection methods outperform filterbased approaches, they commonly suffer potential overfitting to the training data and, hence, decreased generalization ability. Existing filter-based feature selection approaches in the context of media classification employ primarily individual feature evaluation to assess the quality of each feature component. For example, Simmermacher et al. [51] and Deng et al. [8] exploit the performance of information gain, information gain ratio, and symmetrical uncertainty for musical instrument classification. The authors report that the information gain achieves comparable performance to the other two filter-based methods. Similarly, Doraisamy et al. [9] evaluate information gain ratio, Chi-square, correlation-based, and SVM-based feature selection in the context of genre classification of traditional Malay music. The achieved results indicate similar performance of the considered feature selection approaches on the employed dataset. Recently, Huang et al. [22] proposed a self-adaptive harmony search algorithm for the classification of music genres. The authors employ a local feature selection strategy on pairs of music genres which limits the applicability of the approach to scenarios with available prior knowledge about the investigated dataset. Currently, existing approaches and reported evaluations on audio and video classifications are commonly limited to a specific task and a single dataset. In contrast, we explore various application scenarios and provide insights into the selected features using different audio and video datasets. Additionally, we investigate the role of the data and the application scenario on the selected features.

\section{Proposed approach}

In this paper, we propose an unsupervised group feature selection approach which is an extended version of $[45,46]$. The approach exploits the canonical correlations between 
features (of potentially strongly varying dimensionality) to estimate the relevance of every single feature. The assumption is that low-correlated features provide additional or complementary information, while highly correlated features indicate redundant information. Therefore, the inclusion of the latter would only increase the target dimensionality without improving the descriptive power of the resulting feature set.

CCA is a multivariate multiple regression method that measures correlations between multi-dimensional vectors (features) with potentially different number of variables (feature components) [20]. For each of the input vectors, base vectors are generated in a way that optimizes their correlations in terms of mutual information, which serves as an indicator for statistical coherence. The base vectors have maximum dimensions of the minimum dimensionality of the original input vectors and are independent of any affine transformations or the underlying coordinate system. Therefore, CCA allows for the analysis of relationships between multiple dependent or independent variables. For the special case of low-dimensional features, i.e., features that comprise one or two components, we employ the linear and multiple correlation approaches by Hotteling [20].

Let $X$ and $Y$ be two features of (potentially) different sizes: $X=\left[X_{1}, \ldots, X_{p}\right], Y=\left[Y_{1}, \ldots, Y_{q}\right]$. For each feature, there is a solution for the corresponding linear combinations $U=X \alpha$ and $V=Y \beta$. To optimize the correlation coefficient $\rho$ between $U$ and $V$, the vectors $\alpha$ and $\beta$ have to be found such that $\rho$ is maximized, i.e., $\max _{\alpha, \beta} \rho(U, V)$, where $\rho(U, V)$ is given by:

$$
\begin{aligned}
\rho(U, V) & =\frac{\operatorname{Cov}(U, V)}{\sqrt{\operatorname{Var}(U)} \sqrt{\operatorname{Var}(V)}} \\
& =\frac{\operatorname{Cov}(X \alpha, Y \beta)}{\sqrt{\operatorname{Var}(X \alpha)} \sqrt{\operatorname{Var}(Y \beta)}} \\
& =\frac{\alpha^{\prime} \operatorname{Cov}(X, Y) \beta}{\sqrt{\alpha^{\prime} \operatorname{Var}(X) \alpha} \sqrt{\beta^{\prime} \operatorname{Var}(Y) \beta}} .
\end{aligned}
$$

We consider the correlation coefficient, $\rho$, between any two features $X$ and $Y$ as an estimator for their complementarity and, hence, relevance. Initially, we rank all features according to their pairwise correlation coefficients. A high rank is assigned to weakly correlated and thus complementary feature pairs. Strongly correlated and thus redundant feature pairs get low ranks. The feature pair with the lowest correlation builds the initial target feature set. All other features are processed in a loop. In each iteration, an internal evaluation of the correlations of the remaining features to the current target set estimates whether or not a feature contributes additional information to the target set. For this purpose, we consider the current target set as a single multi-dimensional feature and exploit its correlation to every feature in the remaining group feature set. The fea- ture with the lowest correlation is added to the target set if it does not exceed a predefined threshold, $t h_{c}$. The purpose of the threshold is to avoid the consideration of features that have a high correlation value. Each addition to the target set influences the correlations to the remaining features. The more features are included in the target set, the higher the correlations of the target set to the remaining features becomes. Therefore, the underlying CCA is re-initialized after each feature addition. In the original approach, we only computed CCA values once and processed (added) the corresponding features based on this static list. The adaptation of CCA has considerable consequences on the selected features. We will show in the evaluation that the selected features demonstrate extraordinary quality. They will be used throughout the paper for different datasets as well as different tasks and compete with feature sets that were specifically and carefully selected for these datasets and/or tasks in many cases. The increase in the correlations additionally allows for the autonomous termination of the feature selection process. If the lowest correlation between the target set and the remaining features exceeds the predefined threshold, $t h_{c}$, the selection process terminates. Eventually, an additional, optional stopping criterion can be employed to terminate the feature selection process if, for example, a feature set of a certain size is desired. In this work, we do not employ such stopping criterion but investigate all feature pairs to autonomously identify the optimal set of features for the given data. Algorithm 1 illustrates a simplified scheme for the proposed group feature selection approach.

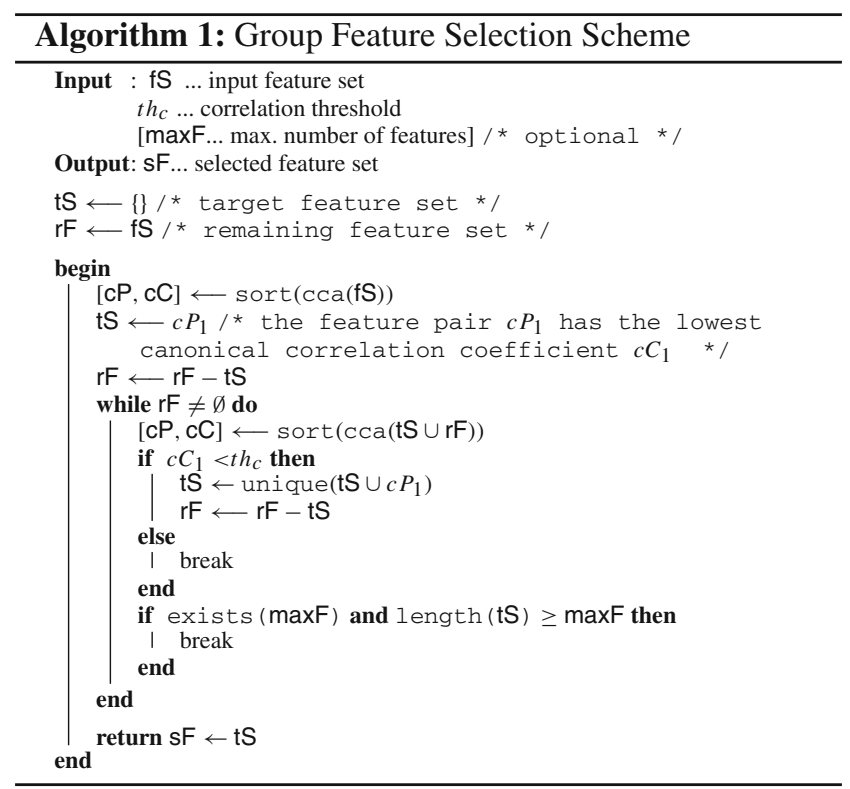


Table 1 Overview of the employed audio datasets

Table 2 Overview of the employed video datasets

\begin{tabular}{llcccc}
\hline Scenario & Dataset & Samples & Classes & & \multicolumn{2}{l}{ Samples/class } \\
\cline { 5 - 6 } & & & Mean $(m)$ & Std $(\sigma)$ \\
\hline Genre & ISMIR [24] & 1458 & 6 & 243 & 209 \\
Instrument & IOWA [23] & 894 & 22 & 41 & 55
\end{tabular}

\begin{tabular}{|c|c|c|c|c|c|c|c|}
\hline \multirow[t]{2}{*}{ Scenario } & \multirow[t]{2}{*}{ Dataset } & \multirow[t]{2}{*}{ Videos } & \multirow[t]{2}{*}{ Classes } & \multirow[t]{2}{*}{ Segments (s) } & \multirow[t]{2}{*}{ Samples } & \multicolumn{2}{|c|}{ Samples/class } \\
\hline & & & & & & Mean $(m)$ & $\operatorname{Std}(\sigma)$ \\
\hline \multirow[t]{3}{*}{ Sub-genre } & $\mathrm{BBC}$ & 9 & 3 & 2 & 16,191 & 5397 & 0.00 \\
\hline & & & & 10 & 3231 & 1077 & 0.00 \\
\hline & & & & 30 & 1071 & 357 & 0.00 \\
\hline \multirow[t]{3}{*}{ Genre } & RAI [37] & 264 & 7 & 2 & 209,300 & 29,900 & 25,635 \\
\hline & & & & 10 & 41,721 & 5960 & 5070 \\
\hline & & & & 30 & 13,791 & 1970 & 1687 \\
\hline
\end{tabular}

Finally, it has to be stated that the proposed approach does not explicitly handle noisy features, which in general exhibit low correlations. For such a case, a preprocessing step (data cleaning) is needed to eliminate potentially noisy features. Such a step is not considered in this work.

\section{Evaluation setup}

\subsection{Application scenarios and datasets}

We investigate two audio classification scenarios: music genre and instrument classification. The two underlying realworld datasets demonstrate different degrees of difficulty in the context of high-dimensional data mining: strongly varying number of samples in comparison with the overall feature dimensionality, number of classes, and class distribution (see Table 1). Additionally, the nature of the data does not allow for any assumptions about the distribution of single feature components or complete group features. The datasets are well established in the domain of audio analysis and, thus, suitable for a comparison with related work.

Additionally, we investigate two video datasets: BBC documentaries and RAI TV broadcasts (see Table 2). The $\mathrm{BBC}$ data is a self-collected set of $9 \mathrm{~h}$ of videos from the BBC's YouTube channel. ${ }^{1}$ It covers three sub-genres: technical, nature, and music. Although the semantic focus of the three sub-genres is strongly varying, all videos in this set are composed, edited, and post-processed in a very similar way, at least from a technical (editorial) point of view. The second video dataset contains more than $100 \mathrm{~h}$ of complete broadcasted programmes of RAI television and compromises 7 genres: commercials, football, music, news,

${ }^{1}$ https://www.youtube.com/user/BBC/. talk shows, weather forecasts, and cartoons [37,38]. In contrast to the BBC documentaries, the RAI broadcasts exhibit strongly varying structures and no explicit regularities among the different genres. As a result, this heterogeneous corpus corresponds to a conventional genre classification task, whereas the $\mathrm{BBC}$ documentaries allow for the investigation of a sub-genre classification scenario. Since the different video datasets are available as different video container files, we first extract the audio tracks and convert them to PCM audio files. The audio tracks are segmented into chunks of 2, 10, and $30 \mathrm{~s}$. This subdivision is carried out with an overlap of $50 \%$ in order to maintain acoustic information near the segmentation boundaries. Especially when considering small segments of the audio signals, passages of constant silence may appear. These segments do not have any expressiveness and may cause errors in the feature extraction process. Therefore, we perform silence detection by means of a noise threshold of $-60 \mathrm{~dB}$ and remove detected silent segments from the dataset. This step has a low impact on the following analysis: none of the segments from the BBC documentaries and only $0.2 \%$ of the $2 \mathrm{~s} \mathrm{seg-}$ ments from the RAI TV dataset are identified as silence and removed.

\subsection{Audio features}

We employ a set of 50 group features (679 feature components in total) that includes representative and comprehensive audio features from the temporal and frequency domains. They represent different audio aspects, such as harmonic structure, rhythm, pitch, and loudness. For more details on all features please refer to [36]. Table 3 provides an overview of the extracted features and the corresponding number of components (feature dimension). 
Table 3 Overview of the employed features (in alphabetical order) and the corresponding dimensions (D)

\begin{tabular}{|c|c|c|}
\hline Feature & Feature name & $\mathrm{D}$ \\
\hline $\mathrm{AD}$ & Amplitude Descriptor & 40 \\
\hline $\mathrm{BFCC}$ & Bark-scale Frequency Cepstral Coefficients & 40 \\
\hline BTHI & Beat Histogram & 7 \\
\hline CRMA & Chroma CENS Features & 24 \\
\hline $\mathrm{E} 4 \mathrm{~Hz}$ & $4 \mathrm{~Hz}$ Modulation Energy & 2 \\
\hline GPD & Group Delay & 40 \\
\hline HMDV & Harmonic Derivate & 16 \\
\hline HZCR & High Zero Crossing Rate & 1 \\
\hline LPC & Linear Predictive Coding & 40 \\
\hline LPCC & Linear Prediction Cepstral Coefficients & 40 \\
\hline LPZC & Linear Prediction ZCR & 2 \\
\hline LSP & Line Spectral Pairs & 40 \\
\hline M7_AFF & MPEG-7 Audio Fundamental Frequency & 4 \\
\hline M7_AH & MPEG-7 Audio Harmonicity & 4 \\
\hline M7_AP & MPEG-7 Audio Power & 2 \\
\hline M7_ASB & MPEG-7 Audio Spectrum Basis & 72 \\
\hline M7_ASC & MPEG-7 Audio Spectrum Centroid & 2 \\
\hline M7_ASF & MPEG-7 Audio Spectrum Flatness & 34 \\
\hline M7_ASP & MPEG-7 Audio Spectrum Projection & 16 \\
\hline M7_ASS & MPEG-7 Audio Spectrum Spread & 2 \\
\hline M7_AW & MPEG-7 Audio Waveform & 4 \\
\hline M7_HSC & MPEG-7 Harmonic Spectral Centroid & 1 \\
\hline M7_HSD & MPEG-7 Harmonic Spectral Deviation & 1 \\
\hline M7_HSS & MPEG-7 Harmonic Spectral Spread & 1 \\
\hline M7_HSV & MPEG-7 Harmonic Spectral Variation & 1 \\
\hline M7_LAT & MPEG-7 Log Attack Time & 1 \\
\hline M7_SC & MPEG-7 Spectral Centroid & 1 \\
\hline MFCC & Mel-scale Frequency Cepstral Coefficients & 40 \\
\hline PLP & Perceptual Linear Prediction & 38 \\
\hline PTCH & Pitch & 2 \\
\hline PTCT & Pitch Contour & 2 \\
\hline PTVB & Pitch Vibration & 1 \\
\hline R_ZC & Range of Zero Crossing Rate & 1 \\
\hline RMS & Root Mean Square & 2 \\
\hline ROFF & Spectral Rolloff & 2 \\
\hline RPLP & Raster PLP & 38 \\
\hline RYPT & Rhythm Patterns & 20 \\
\hline SBER & Subband Energy Ratio & 10 \\
\hline SF & Spectral Flux & 2 \\
\hline SONE & Loudness & 40 \\
\hline SPCR & Spectral Crest & 8 \\
\hline SPCT & Spectral Center & 2 \\
\hline SPDI & Spectral Dispersion & 2 \\
\hline SPEY & Spectral Entropy & 8 \\
\hline SPPS & Spectral Peak Structure & 2 \\
\hline SPRE & Spectral Renyi Entropy & 8 \\
\hline
\end{tabular}

Table 3 continued

\begin{tabular}{llr}
\hline Feature & Feature name & D \\
\hline SPSL & Spectral Slope & 8 \\
STE & Short Time Energy & 2 \\
VDR & Volume Dynamic Range & 1 \\
ZCR & Zero Crossing Rate & 2 \\
& & 679 \\
\hline
\end{tabular}

\subsection{Performance metrics}

We consider two performance metrics: weighted F-score for measuring the classification accuracy and robustness for measuring the reliability of a feature selection method to repeatedly select the same feature set for a given application scenario in different runs.

The weighted $F$-score accounts for the class distribution of the underlying dataset by building the arithmetic mean of the standard $F$-score values for the individual classes:

$F_{\beta}^{w}=\frac{1}{n} \sum_{c \in C} F_{\beta}(c) \times n_{c}$,

where $n_{c}$ denotes the number of instances per class $c, n$ is the number of instances in total, and $F_{\beta}$ is the standard $F$-score:

$F_{\beta}=\left(1+\beta^{2}\right) \frac{\text { precision } \times \text { recall }}{\beta^{2} \times \text { precision }+ \text { recall }}$

Up to now robustness (stability) has not been considered to be an issue in the domain of media analysis and media mining. This paper is one of the first to draw the attention to the fact that in dynamic environments and continuously changing large media repositories, robustness should be an important aspect of feature selection. Robust feature selection has been addressed in other domains, such as in internet traffic anomaly detection [41] and, especially, in bioinformatics. Several approaches have been suggested for the analysis of gene expression data obtained from microarray experiments [1,4], for example in $[19,39,44,58]$. This challenging problem deals with a feature space of at least tens of thousands of genes, a small sample size, and noise and variability due to the experimental setup making the robustness of selected features an important goal.

Robustness measures are usually based on the similarity of feature sets. An approach producing very similar feature sets in different runs or in different tasks is considered to be robust. A very popular measure for set similarity in statistics is the Jaccard index defined as the cardinality of the intersection divided by the cardinality of the union of two (or more) sets [31]. Additional measures have been introduced in the literature, and an overview and comparison is provided by 
Table 4 Influence of the correlation threshold $\left(t h_{c}\right)$ on the classification accuracy in terms of F1-scores (F1), number $(\mathrm{N})$ of selected features, and dimensionality (D) of the corresponding feature subset in comparison to the full feature set (Full FS) for the employed audio datasets

\begin{tabular}{|c|c|c|c|c|c|c|c|c|c|c|}
\hline \multirow[t]{3}{*}{$t h_{c}$} & \multicolumn{5}{|c|}{ ISMIR } & \multicolumn{5}{|c|}{ IOWA } \\
\hline & \multicolumn{3}{|c|}{ Features } & \multicolumn{2}{|l|}{ F1 } & \multicolumn{3}{|c|}{$\underline{\text { Features }}$} & \multicolumn{2}{|l|}{$\underline{F 1}$} \\
\hline & $\mathrm{D}$ & $(\%)$ & $\mathrm{N}$ & KNN & SVM & $\mathrm{D}$ & $(\%)$ & $\mathrm{N}$ & $\mathrm{KNN}$ & SVM \\
\hline 0.95 & 41 & $(6 \%)$ & 17 & 0.6942 & 0.7504 & 29 & $(4 \%)$ & 16 & 0.8600 & 0.9181 \\
\hline 0.96 & 43 & $(6 \%)$ & 18 & 0.6901 & 0.7413 & 31 & $(5 \%)$ & 17 & 0.8682 & 0.9252 \\
\hline 0.97 & 51 & $(8 \%)$ & 19 & 0.7011 & 0.7637 & 36 & $(5 \%)$ & 18 & 0.8904 & 0.9340 \\
\hline 0.98 & 69 & $(10 \%)$ & 20 & 0.7077 & 0.7746 & 37 & $(5 \%)$ & 19 & 0.9020 & 0.9432 \\
\hline 0.99 & 88 & $(13 \%)$ & 23 & 0.7171 & 0.7923 & 47 & $(7 \%)$ & 21 & 0.9045 & 0.9449 \\
\hline 1.00 & 143 & $(21 \%)$ & 34 & 0.7360 & 0.8175 & 82 & $(12 \%)$ & 29 & 0.9084 & 0.9522 \\
\hline Full FS & 679 & $(100 \%)$ & 50 & 0.7720 & 0.8550 & 679 & $(100 \%)$ & 50 & 0.9440 & 0.9780 \\
\hline
\end{tabular}

Somol and Novovicova [53]. We principally follow the definition of the Jaccard index. However, we aim at making differences between approaches larger and more expressive by not using the cardinality of the union but the cardinality of the smaller set as divisor. We therefore measure robust$n e s s, R$, of a feature selection approach by considering the co-occurrences of the selected features in a final feature set, $f S$, averaged over a predefined number of independent and randomly initialized runs, $r$, as follows:

$R=\frac{2}{r(r-1)} \sum_{i=1}^{r-1} \sum_{j=i+1}^{r} \frac{\left|f S_{i} \cap f S_{j}\right|}{\min \left(\left|f S_{i}\right|,\left|f S_{j}\right|\right)}$

\subsection{Classifiers}

We compare the performance of two well-established classifiers for audio classification [14]: K-nearest neighbor (KNN) [6], and support vector machine (SVM) [56]. In previous work, we additionally considered multinomial logistic regression (MLG) and random forest (RF) [5] in various audio-based scenarios [46]. Both MLG and RF classifiers demonstrated comparable performance to KNN and SVM at notably higher computational costs. Therefore, in this work we focus on the performance of KNN and SVM only. The classifier parameters have been selected based on preliminary experiments on all investigated datasets with respect to classification accuracy and runtime performance. We employ $\mathrm{KNN}$ with $k=1$ and the Euclidean distance with no distance weighting. The SVM uses a linear kernel function. All classifications are randomly initialized, 10 -fold cross-validated with respect to the underlying class distribution, and run 10 times independently.

\section{Evaluation results}

\subsection{Correlation threshold}

In our first experiment, we elaborate the influence of the correlation threshold, $t h_{c}$, on the selected feature set (both in terms of dimensionality and number of selected features) and the resulting classification performance in terms of $F 1$-score. Additionally, we put the results in relation to the performance of the full feature set. For this experiment, we perform feature selection on $10 \%$ of the corresponding data in 10 randomly initialized runs. For the classification, each run is 10 -fold cross-validated. Tables 4 and 5 summarize the achieved results averaged over the 10 independent runs for different correlation thresholds, $t h_{c}=\{0.95,0.96, \ldots, 1.00\}$, for the audio and video sets, respectively. The results show that an increase in the correlation threshold leads to an increase in both the dimensions of the selected feature set and the classification accuracy. This monotonic increase can be observed independently of the underlying classifier and dataset. Noteworthy is the remarkable reduction in feature dimensionality at a comparable performance level. For example, the BBC data with video segments of $2 \mathrm{~s}$ achieves an F1-score of $99.90 \%$ using the SVM classifier. The employment of the proposed feature selection approach with a correlation threshold of 0.95 achieves a notable reduction in the feature set to $28 \%$ of the full feature set at highly comparable classification performance indicated by the F1-score of $99.81 \%$. The reduction in feature dimensionality leads to lower computational costs. Moreover, a lower feature set dimensionality supports the application of methods which cannot handle high-dimensional data.

The results additionally show a notable difference between the performance across the different segment sizes for the two video datasets. BBC performs better for smaller segments (e.g., F1-score of $99.81 \%$ for audio segments of size $2 \mathrm{~s}$ and $\mathrm{F} 1$-score of $92.81 \%$ for segments of size $30 \mathrm{~s}$ using the SVM classifier and $\left.t h_{c}=0.95\right)$. On the opposite side, the RAI dataset performs slightly better for increasing segment sizes (e.g., F1-score of $80.62 \%$ for segments of size $2 \mathrm{~s}^{2}$ and F1-score of $90.83 \%$ for segments of size $30 \mathrm{~s}$, again using the SVM classifier and $t h_{c}=0.95$ ). This inverse tendency is

\footnotetext{
2 Please note that due to computational reasons we performed classification on $25 \%$ of the RAI dataset with segments of size $2 \mathrm{~s}$ as an approximation for the overall performance.
} 
Table 5 Influence of the correlation threshold $\left(t h_{c}\right)$ on the classification accuracy in terms of F1-scores (F1), number $(\mathrm{N})$ of selected features, and dimensionality (D) of the corresponding feature subset in comparison to the full feature set (Full FS) for the employed video datasets

\begin{tabular}{|c|c|c|c|c|c|c|c|c|c|c|c|c|c|c|c|}
\hline \multirow[t]{3}{*}{$t h_{c}$} & \multicolumn{5}{|l|}{$2 \mathrm{~s}$} & \multicolumn{5}{|l|}{$10 \mathrm{~s}$} & \multicolumn{5}{|l|}{$30 \mathrm{~s}$} \\
\hline & \multicolumn{3}{|c|}{$\underline{\text { Features }}$} & \multicolumn{2}{|l|}{$\mathrm{F} 1$} & \multicolumn{3}{|c|}{$\underline{\text { Features }}$} & \multicolumn{2}{|l|}{$\underline{F 1}$} & \multicolumn{3}{|c|}{$\underline{\text { Features }}$} & \multicolumn{2}{|l|}{$\mathrm{F} 1$} \\
\hline & $\mathrm{D}$ & $(\%)$ & $\mathrm{N}$ & KNN & SVM & $\mathrm{D}$ & $(\%)$ & $\mathrm{N}$ & $\mathrm{KNN}$ & SVM & $\mathrm{D}$ & $(\%)$ & $\mathrm{N}$ & KNN & SVM \\
\hline \multicolumn{16}{|l|}{$B B C$} \\
\hline 0.95 & 191 & $(28 \%)$ & 26 & 0.9964 & 0.9981 & 113 & $(17 \%)$ & 22 & 0.9701 & 0.9858 & 49 & $(7 \%)$ & 20 & 0.9505 & 0.9281 \\
\hline 0.96 & 208 & $(31 \%)$ & 26 & 0.9974 & 0.9989 & 127 & $(19 \%)$ & 23 & 0.9704 & 0.9877 & 56 & $(8 \%)$ & 21 & 0.9504 & 0.9424 \\
\hline 0.97 & 221 & $(32 \%)$ & 28 & 0.9970 & 0.9980 & 140 & $(21 \%)$ & 24 & 0.9733 & 0.9896 & 61 & $(9 \%)$ & 22 & 0.9534 & 0.9534 \\
\hline 0.98 & 254 & $(37 \%)$ & 31 & 0.9974 & 0.9984 & 156 & $(23 \%)$ & 27 & 0.9824 & 0.9905 & 67 & $(10 \%)$ & 23 & 0.9515 & 0.9549 \\
\hline 0.99 & 360 & $(53 \%)$ & 35 & 0.9980 & 0.9990 & 182 & $(27 \%)$ & 29 & 0.9888 & 0.9937 & 76 & $(11 \%)$ & 25 & 0.9606 & 0.9657 \\
\hline 1.00 & 679 & $(100 \%)$ & 50 & 0.9990 & 0.9990 & 316 & $(47 \%)$ & 41 & 0.9958 & 0.9977 & 104 & $(15 \%)$ & 32 & 0.9709 & 0.9767 \\
\hline Full FS & 679 & $(100 \%)$ & 50 & 0.9990 & 0.9990 & 679 & $(100 \%)$ & 50 & 0.9970 & 0.9980 & 679 & $(100 \%)$ & 50 & 0.9910 & 0.9940 \\
\hline \multicolumn{16}{|l|}{$R A I$} \\
\hline 0.95 & 175 & $(26 \%)$ & 26 & 0.7709 & 0.8062 & 103 & $(15 \%)$ & 20 & 0.8725 & 0.8741 & 103 & $(15 \%)$ & 20 & 0.9232 & 0.9083 \\
\hline 0.96 & 178 & $(26 \%)$ & 27 & 0.7743 & 0.8101 & 124 & $(18 \%)$ & 21 & 0.8808 & 0.8758 & 124 & $(18 \%)$ & 21 & 0.9302 & 0.9142 \\
\hline 0.97 & 192 & $(28 \%)$ & 29 & 0.8016 & 0.8282 & 146 & $(22 \%)$ & 23 & 0.8954 & 0.8859 & 146 & $(22 \%)$ & 23 & 0.9347 & 0.9194 \\
\hline 0.98 & 223 & $(33 \%)$ & 31 & 0.8206 & 0.8369 & 170 & $(25 \%)$ & 27 & 0.9047 & 0.8946 & 170 & $(25 \%)$ & 27 & 0.9504 & 0.9360 \\
\hline 0.99 & 242 & $(36 \%)$ & 34 & 0.8246 & 0.8499 & 283 & $(42 \%)$ & 31 & 0.9177 & 0.9256 & 283 & $(42 \%)$ & 31 & 0.8795 & 0.9415 \\
\hline 1.00 & 679 & $(100 \%)$ & 50 & 0.9167 & 0.9183 & 679 & $(100 \%)$ & 50 & 0.9820 & 0.9690 & 679 & $(100 \%)$ & 50 & 0.9790 & 0.9800 \\
\hline Full FS & 679 & $(100 \%)$ & 50 & 0.9167 & 0.9183 & 679 & $(100 \%)$ & 50 & 0.9820 & 0.9690 & 679 & $(100 \%)$ & 50 & 0.9790 & 0.9800 \\
\hline
\end{tabular}

primarily due to the substantial difference in the nature of the underlying data. While the BBC data are very homogeneous (all documentaries share common structure and elements), the RAI data are distinctive to a certain degree. As a result, the $\mathrm{BBC}$ set requires higher granulated segments to capture more descriptive information and, thus, better distinguish across the different sub-genres of documentaries.

The comparison of the two classifiers, KNN and SVM, indicates existing data dependency of the overall performance in terms of F1-score. While for the audio datasets SVM notably outperforms KNN, for the video datasets the performance difference vanishes. In general, SVM outperforms KNN. However, in very specific data settings (cp. RAI dataset, segment size of $30 \mathrm{~s}, t h_{c}=\{0.95,0.96,0.97,0.98\}$ ) KNN demonstrates superior performance over SVM at significantly lower computation costs.

Finally, we investigate the robustness of the proposed feature selection approach, i.e., its ability to select the same features in different runs for the same dataset. Figure 1 shows the achieved results for all datasets for the previously considered threshold settings. The results indicate two trends. First, the larger the available data, the more robust is the selected feature set. For example, the RAI dataset with segments of $2 \mathrm{~s}$ (209.300 samples in total) achieves an average intersection of $98.46 \%$ in contrast to the same dataset with segments of $30 \mathrm{~s}$ (13.791 samples in total) which achieves an average intersection of $85.57 \%$ for $t h_{c}=0.95$. This tendency can be observed over the different threshold settings and for the three variations of the BBC dataset as well. Second, the higher the correlation threshold, the more robust the selected feature set. For example, the ISMIR dataset achieves $80.65 \%$ feature overlapping for $t h_{c}=0.95$ and $97.37 \%$ for $t h_{c}=1.00$. This tendency is closely related to the number of selected features and, again, independent of the underlying dataset. Overall, the average intersection over all datasets and threshold settings is $92 \%$ indicating the high robustness of the proposed feature selection approach.

\subsection{Data size for feature selection}

In this experiment, we investigate the influence of the size of available data on the feature selection process (in terms of dimensionality and number of the selected features and the robustness of the resulting feature sets over different runs on the same data) and on the classification performance (in terms of F1-scores). For this evaluation, we consider the two audio datasets, ISMIR and IOWA, and two video datasets, $\mathrm{BBC}$ and RAI, segments of size $30 \mathrm{~s}$. We fix the correlation threshold, $t h_{c}$, to 0.99 and consider different portion sizes of the investigated datasets for the feature selection process $(1,5,10,20,50$, and $100 \%)$ in 10 randomly initialized and 10 -fold cross-validated runs. The smallest class in the IOWA dataset is of size 9; therefore, the lowest reported portion for this dataset is $10 \%$.

Table 6 summarizes the results in terms of dimensionality of the selected feature sets and the corresponding classi- 




Fig. 1 Overlapping of the selected features over the 10 independent and randomly initialized runs for the different datasets and threshold settings

Table 6 Influence of the size of the data available for feature selection on the classification accuracy in terms of F1-scores $(\mathrm{F} 1)$, number $(\mathrm{N})$ of selected features, and dimensionality (D) of the corresponding feature subset in comparison to the full feature set (Full FS) for the investigated datasets

\begin{tabular}{|c|c|c|c|c|c|c|c|c|c|c|}
\hline \multicolumn{11}{|c|}{ Audio datasets } \\
\hline \multirow[t]{3}{*}{ Data } & \multicolumn{5}{|c|}{ ISMIR } & \multicolumn{5}{|c|}{ IOWA } \\
\hline & \multicolumn{3}{|c|}{ Features } & \multicolumn{2}{|l|}{ F1 } & \multicolumn{3}{|c|}{ Features } & \multicolumn{2}{|l|}{$\mathrm{F} 1$} \\
\hline & $\mathrm{D}$ & $(\%)$ & $\mathrm{N}$ & KNN & SVM & $\mathrm{D}$ & $(\%)$ & $\mathrm{N}$ & KNN & SVM \\
\hline $1 \%$ & 11 & $(2 \%)$ & 9 & 0.5986 & 0.6057 & - & - & - & - & - \\
\hline $5 \%$ & 45 & $(7 \%)$ & 20 & 0.7007 & 0.7727 & - & - & - & - & - \\
\hline $10 \%$ & 88 & $(13 \%)$ & 23 & 0.7171 & 0.7923 & 47 & $(7 \%)$ & 21 & 0.9045 & 0.9449 \\
\hline $20 \%$ & 135 & $(20 \%)$ & 26 & 0.7250 & 0.8029 & 97 & $(14 \%)$ & 24 & 0.8971 & 0.9506 \\
\hline $50 \%$ & 199 & $(29 \%)$ & 29 & 0.7453 & 0.8255 & 234 & $(34 \%)$ & 25 & 0.8757 & 0.9415 \\
\hline $100 \%$ & 225 & $(33 \%)$ & 32 & 0.7560 & 0.8310 & 276 & $(41 \%)$ & 29 & 0.8990 & 0.9520 \\
\hline Full FS & 679 & $(100 \%)$ & 50 & 0.7720 & 0.8550 & 679 & $(100 \%)$ & 50 & 0.9440 & 0.9780 \\
\hline \multicolumn{11}{|c|}{$\underline{\text { Video datasets }}$} \\
\hline \multirow[t]{3}{*}{ Data } & \multicolumn{5}{|c|}{ BBC-30 s } & \multicolumn{5}{|c|}{ RAI-30 s } \\
\hline & \multicolumn{3}{|c|}{ Features } & \multicolumn{2}{|l|}{$\mathrm{F} 1$} & \multicolumn{3}{|c|}{ Features } & \multicolumn{2}{|l|}{$\mathrm{F} 1$} \\
\hline & $D$ & $(\%)$ & $\mathrm{N}$ & $\overline{\mathrm{KNN}}$ & SVM & $\overline{\mathrm{D}}$ & $(\%)$ & $\mathrm{N}$ & $\overline{\mathrm{KNN}}$ & SVM \\
\hline $1 \%$ & 8 & $(1 \%)$ & 7 & 0.8281 & 0.6567 & 80 & $(12 \%)$ & 23 & 0.9276 & 0.9090 \\
\hline $5 \%$ & 42 & $(6 \%)$ & 25 & 0.9637 & 0.9314 & 240 & $(35 \%)$ & 30 & 0.9094 & 0.9420 \\
\hline $10 \%$ & 76 & $(11 \%)$ & 25 & 0.9606 & 0.9657 & 283 & $(42 \%)$ & 31 & 0.8795 & 0.9415 \\
\hline $20 \%$ & 145 & $(21 \%)$ & 27 & 0.9701 & 0.9802 & 328 & $(48 \%)$ & 32 & 0.9089 & 0.9562 \\
\hline $50 \%$ & 301 & $(44 \%)$ & 31 & 0.9690 & 0.9930 & 320 & $(47 \%)$ & 35 & 0.9122 & 0.9575 \\
\hline $100 \%$ & 357 & $(53 \%)$ & 33 & 0.9810 & 0.9930 & 340 & $(50 \%)$ & 33 & 0.9120 & 0.9580 \\
\hline Full FS & 679 & $(100 \%)$ & 50 & 0.9910 & 0.9940 & 679 & $(100 \%)$ & 50 & 0.9790 & 0.9800 \\
\hline
\end{tabular}

fication performance. The results show that an increasing size of available data for feature selection usually leads to a higher number of selected features. This is due to the fact that more data usually reveals more aspects about the underlying data characteristics. Therefore, a larger number of features is required to capture more descriptive information. However, while the amount of data for the feature selection is rapidly growing, the size of the selected feature set only displays a slight increase. This indicates that, at some point no additional information is introduced by newly added instances. Overall, the size of the selected feature set is again notably lower than the size of the full feature set.
Figure 2 shows the robustness of the selected feature sets over the different runs for each of the investigated datasets. The results reflect the previous observations. The random selection of a low portion of data (e.g., 1-5\%) may potentially lead to the selection of data subsets that capture very differing characteristics resulting in a (relatively) low intersection of the selected feature sets. In contrast, the probability that a larger data subset will account for a greater deal of the characteristics of the underlying data is significantly higher. Therefore, the more data are available for the feature selection process, the higher the robustness of the selected feature set. Nevertheless, the results show that performing feature 




Fig. 2 Overlapping of the selected features over the 10 independent and randomly initialized runs for varying percentages of available data for feature selection for the investigated datasets

selection on $10 \%$ of the data already achieves highly robust feature sets with an intersection of approx. $90 \%$ over the different randomly initialized runs.

\subsection{Comparison to related work on feature selection}

In this section, we compare the performance of the proposed group feature selection approach with our previous work, CcaFS [45], which applies CCA on feature pairs, with two well-established but supervised subset-based feature selection approaches, consistency-based (ConFS) [34] and correlation-based(CorrFS) [16], and with the group feature selection approach group lasso (GL) [62]. For all approaches, we perform feature selection and model building for the classifier on $20 \%$ of the available data. The performance on the remaining data is evaluated in terms of both F1-score and robustness of the selected feature sets averaged over 10 independent and randomly initialized runs. For the classification, we employ the SVM classifier and perform 10-fold crossvalidation of each run.

Table 7 condenses the results for the four data sets, ISMIR, IOWA, BBC (30 s) and RAI (30 s), in terms of the selected feature set dimension (D), the percentage of compression in comparison to the initial (full) feature set (\%), F1-score using the SVM classifier, and robustness (R). The results show no overall winner over all four datasets in terms of F1-score: Group lasso achieves the best F1-score for BBC and RAI, and the supervised correlation-based approach the best results for ISMIR and IOWA. Our approach is second for three datasets and third for IOWA, however, with very small differences to the best approaches. Robustness shows an entirely different picture: Our approach is best in three cases, but usually with large differences to the other methods. The number of features selected is largest for our first approach CcaFS in three cases. A comparison of our new and our first approaches shows that we could improve F1-scores and robustness and at the same time reduce the number of selected features. Only in the case of the IOWA dataset, robustness could not be improved. Overall, we demonstrated that our new, unsupervised approach yields comparable results in terms of F1-score to the other approaches while at the same time yielding considerably better robustness.

\subsection{Comparison to related work on media classification}

In this section, we present a comparison of the results achieved by the proposed approach with related works reporting results on the same three publicly available datasets: ISMIR (music genre classification), IOWA (musical instrument identification), and RAI (TV genre classification).

For the first ISMIR music dataset, we rerun the experiments with the same settings as defined by the ISMIR 2004 genre classification contest $\mathrm{t}^{3}$ in order to provide for comparability. In the contest, the classification performance is evaluated based on predefined training and test sets (consisting of 729 samples each) using weighted classification accuracy:

$C A=\sum_{c \in \text { genres }} p_{c} C A_{c}$

where $p_{c}$ is the probability of appearance of genre $\mathrm{c}$ and $C A_{c}$ the classification accuracy for $c$. Table 8 provides a summary of most recent works reporting top results on the ISMIR dataset. In general, research in the context of music genre classification is highly tailored to the characteristics of the specific task. For example, Lee et al. [30] elaborate long-term modulation spectral analysis of spectral and cesptral feature trajectories to describe the time-varying behavior of music signals. The authors investigate octave-based spectral contrast (OSC), normalized audio spectrum envelope (NASE), and MFCC features. Overall, the listed approaches exhibit a broad variety in the dimensionality of the considered feature sets. For example, Seo and Lee [48] employ higher-

\footnotetext{
${ }^{3} \mathrm{http}: / /$ ismir2004.ismir.net/genre_contest.
} 
Table 7 Comparison to related feature selection algorithms in terms of number $(\mathrm{N})$ of selected features and their dimensionality (D), F1-scores using the SVM classifier, and robustness (R) of the corresponding feature selection approach

\begin{tabular}{|c|c|c|c|c|c|c|c|c|c|c|}
\hline \multicolumn{11}{|c|}{ Audio datasets } \\
\hline \multirow[t]{3}{*}{ Approach } & \multicolumn{5}{|c|}{ ISMIR } & \multicolumn{5}{|c|}{ IOWA } \\
\hline & \multicolumn{3}{|c|}{ Features } & \multirow[t]{2}{*}{$\mathrm{F} 1$} & \multirow[t]{2}{*}{$\mathrm{R}$} & \multicolumn{3}{|c|}{ Features } & \multirow[t]{2}{*}{$\mathrm{F} 1$} & \multirow[t]{2}{*}{$\mathrm{R}$} \\
\hline & $\mathrm{D}$ & $(\%)$ & $\mathrm{N}$ & & & $\mathrm{D}$ & $(\%)$ & $\mathrm{N}$ & & \\
\hline ConFS [34] & 10 & $(1 \%)$ & 9 & 0.6540 & 0.0585 & 4 & $(1 \%)$ & 4 & 0.6602 & 0.1278 \\
\hline CorrFS [16] & 69 & $(10 \%)$ & 26 & 0.8118 & 0.3593 & 88 & $(13 \%)$ & 31 & 0.9685 & 0.4399 \\
\hline GL [62] & 44 & $(6 \%)$ & 11 & 0.7582 & 0.5508 & 85 & $(13 \%)$ & 13 & 0.9620 & 0.8545 \\
\hline CcaFS [45] & 122 & $(18 \%)$ & 28 & 0.7927 & 0.8524 & 82 & $(12 \%)$ & 25 & 0.9426 & 0.8654 \\
\hline This work & 135 & $(20 \%)$ & 26 & 0.8029 & 0.9054 & 97 & $(14 \%)$ & 24 & 0.9506 & 0.8108 \\
\hline \multicolumn{11}{|l|}{ Video datasets } \\
\hline \multirow[t]{3}{*}{ Approach } & \multicolumn{5}{|c|}{$\underline{\mathrm{BBC}-30 \mathrm{~s}}$} & \multicolumn{5}{|c|}{$\underline{\text { RAI-30 s }}$} \\
\hline & \multicolumn{3}{|c|}{ Features } & $\mathrm{F} 1$ & $\mathrm{R}$ & \multicolumn{3}{|c|}{ Features } & $\mathrm{F} 1$ & $\mathrm{R}$ \\
\hline & $\mathrm{D}$ & $(\%)$ & $\mathrm{N}$ & & & $\mathrm{D}$ & $(\%)$ & $\mathrm{N}$ & & \\
\hline ConFS [34] & 3 & $(<1 \%)$ & 3 & 0.7523 & 0.2593 & 8 & $(1 \%)$ & 7 & 0.7698 & 0.1960 \\
\hline CorrFS [16] & 42 & $(6 \%)$ & 17 & 0.9448 & 0.4907 & 105 & $(16 \%)$ & 27 & 0.9464 & 0.8252 \\
\hline GL [62] & 134 & $(20 \%)$ & 17 & 0.9898 & 0.7237 & 425 & $(63 \%)$ & 26 & 0.9797 & 0.9659 \\
\hline Cca-FS [45] & 121 & $(18 \%)$ & 30 & 0.9749 & 0.8869 & 248 & $(37 \%)$ & 34 & 0.9479 & 0.9138 \\
\hline This work & 145 & $(21 \%)$ & 27 & 0.9802 & 0.9703 & 328 & $(48 \%)$ & 32 & 0.9562 & 0.9771 \\
\hline
\end{tabular}

order moments of short-time spectral features building a 72-dimensional feature vector and achieving a classification accuracy of $84.64 \%$. On the opposite side, Seyerlehner et al. [49] employ block-based features of dimensionality 9448 leading to a classification accuracy of $88.27 \%$. Although, our approach is outperformed by approximately $10 \%$ by Lee et al. [30], it is the only approach that autonomously selects features to represent the provided data. In contrast, Baniya et al. [2], for example, manually omit features based on the standard deviation of the considered features in the very specific data settings. Overall, the lower result by our approach is probably caused by the initial set of 50 features which consists of fundamental and representative audio features only, describing a broad range of general audio characteristics. This implies that we are not employing any task-specific analysis or classification as in the work by Lee et al. [30], for example.

Table 9 summarizes reported results of related approaches on the IOWA dataset. A direct comparison of the results and the underlying approaches is not possible given the major differences in the employed data and the experimental settings. For example, Benetos et al. [3] report top performance on the IOWA dataset in terms of F1-score of $95.06 \%$. However, the authors employ only a small subset of the available data consisting of a well-defined selection of 6 instruments: piano, bassoon, cello, flute, sax, and violin. In contrast, the works by Deng et al. [8] and Simmermacher et al. [51] employ nearly the same dataset size and only omit two instruments: guitar and marimba. Still, the authors simplify the experi-
Table 8 Comparison to related works on the ISMIR dataset (in alphabetical order) in terms of considered feature dimensionality (D) and the resulting weighted classification accuracy $(\mathrm{CA})$

\begin{tabular}{llll}
\hline Approach & Classifier & D & CA \\
\hline Baniya et al. [2] & ELM [21] & 144 & 0.8646 \\
Jang and Jang [25] & SVM & 168 & 0.8340 \\
Lee et al. [30] & LDA [10] & n.a. & 0.8683 \\
Lim et al. [32] & SVM & 468 & 0.8990 \\
Seyerlehner et al. [49] & SVM & 9448 & 0.8827 \\
Seo and Lee [48] & SVM & 72 & 0.8464 \\
This work & SVM & 334 & 0.7792 \\
\hline
\end{tabular}

mental settings and employ the same number of samples for the instruments within each category. In our experiments, we keep the original distribution of samples which provides for a notably imbalanced data setting. Table 10 shows the confusion matrix of the individual instrument classification using 10-fold cross-validation; feature selection is performed using the full dataset with $t h_{c}=0.99$. In general, our results confirm the findings by Deng et al. [8] and Simmermacher et al. [51]. The best classification accuracies are achieved by the piano/others and percussion categories with $100 \%$, followed by the string instruments with $99 \%$. Worst average classifications accuracy is achieved within the woodwind category by samples of bass flute classified as alto flute (and partly vice versa). In contrast to Deng et al. [8] and Simmermacher et al. [51], we identify the brass category as the one with the lowest average classification accuracy of $78 \%$. Overall, our 
Table 9 Comparison to related works (in alphabetical order) on the IOWA dataset with respect to the considered classes/instruments and the resulting classification accuracy in terms of F1-scores (F1)

\begin{tabular}{llll}
\hline Approach & Classes/instruments & Dataset size & F1 \\
\hline Benetos et al. [3] & 6 & 300 & 0.9506 \\
Deng et al. [8] & 20 & 761 & 0.8690 \\
Nielsen et al. [40] & 17 & n.a. & 0.7590 \\
Simmermacher et al. [51] & 20 & 761 & 0.8925 \\
This work & 22 & 894 & \\
10\% FS & & & 0.9449 \\
100\% FS & & & 0.9520
\end{tabular}

FS denotes the percentage of data employed for the feature selection with $t h_{c}=0.99$

Table 10 IOWA confusion matrix of 10 -fold cross-validation (in percentage)

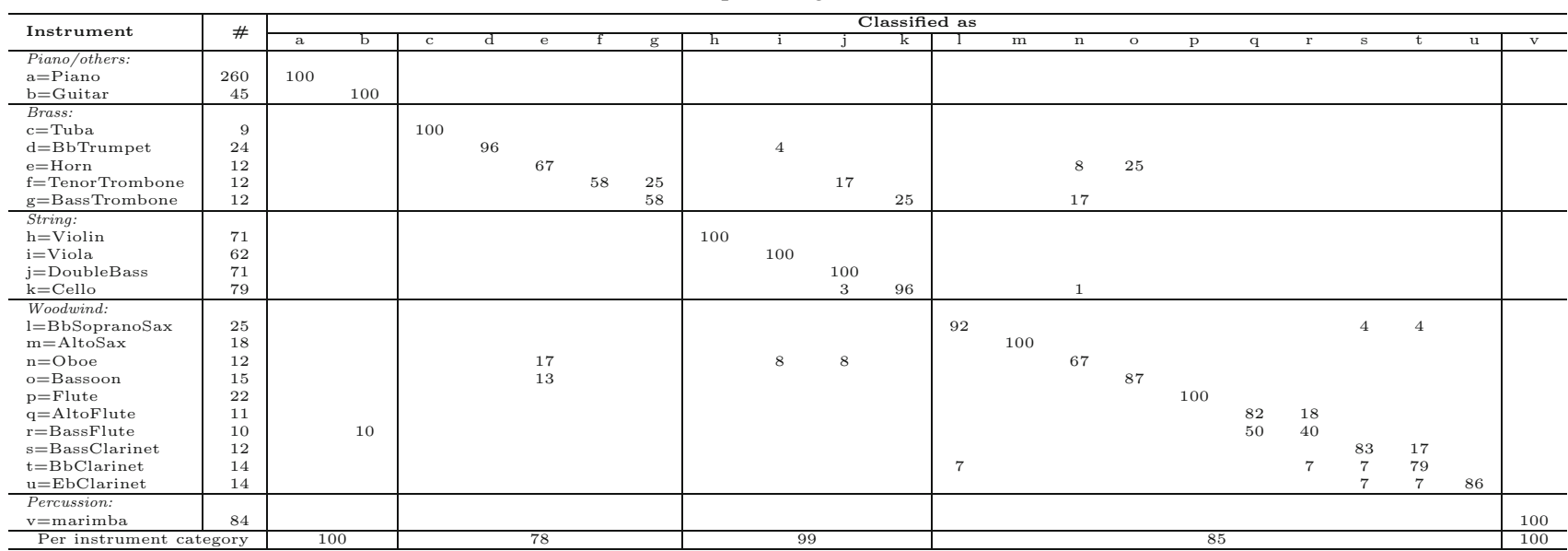

Feature selection (FS) is performed using the full dataset with $t h_{c}=0.99$. \# denotes the number of samples for each instrument. The last line shows the weighted average classification rate per instrument category

proposed approach outperforms previous works in terms of F1-score of $95.20 \%$.

Eventually, we compare our approach with related works reporting evaluation results on the RAI dataset (see Table 11). ${ }^{4}$ In contrast to the previous audio sets, the RAI video dataset allows for the development and comparison of approaches using different modalities. Recent works demonstrate a tendency toward multimodal approaches $[11,37,38]$. The results achieved by our approach indicate the outstanding performance of the selected audio features in terms of F1-score of $95.80 \%$ despite the use of single modality only. Additionally, our approach demonstrates strong competitiveness to the top reported performance by Ekenel and Semela [11]. In addition to some acoustic features, Ekenel and Semena consider visual, structural, and cognitive features. Similar to the works by Montagnuolo et al. [37,38], the features are selected in a way which reflects the editors process in TV production and cannot be applied to arbitrary data. The audio-based approach by Ekenel and Semela [11] achieves comparable performance in terms of F1-score of $95.70 \%$.

\footnotetext{
${ }^{4}$ Please note that the information on the number of videos and their duration in the table may partially differ from the information in the referenced works due to corrected rounding or computation errors.
}

However, the approach is supervised and relies on available data in order to train a SVM model for each genre separately using manually selected features. In contrast, our approach autonomously selects the features that are relevant for the provided dataset and it is not bound to a specific application or dataset. The achieved performance demonstrates the quality of the selected features while at the same time the exploration of a single modality notably reduces the computational effort.

\subsection{Data sensitivity}

Feature selection is commonly done in a very specific context: for a particular application scenario or even for a specific data setting. An initial investigation of the selected feature sets for the considered datasets revealed a high degree of overlapping, i.e., $81 \%$ between the selected features for the audio datasets ISMIR and IOWA and even $90 \%$ for the video datasets BBC and RAI (30 s segments). This is noteworthy since the datasets target different application scenarios. One might argue that the remaining 10 or $19 \%$ would be crucial for the classification performance in the corresponding settings. The alternative hypothesis is that a 
Table 11 Comparison to related works (in alphabetical order) on the RAI dataset in terms of considered modalities: audio (A), visual (V), structural (S), cognitive(C), and the resulting classification accuracy in terms of F1-scores (F1)

Table 12 Evaluation of the classification performance of feature selection on a related dataset

\begin{tabular}{lllll}
\hline Approach & Modality & Videos & Duration (in min) & F1 \\
\hline Ekenel and Semela [11] & A, V, S, C & 262 & 4,164 & 0.9920 \\
Ekenel and Semela [11] & A & 262 & 4,164 & 0.9570 \\
Kim et al. [27] & A & 262 & 4,164 & 0.9430 \\
Montagnuolo et al. [37] & A, V, S, C & n.a. & 6,692 & 0.9200 \\
Montagnuolo et al. [38] & A, V, S, C & 396 & 6,672 & 0.9490 \\
Montagnuolo et al. [38] & A & 396 & 6,672 & 0.7870 \\
Sargent et al. [47] & A, V, S & 264 & n.a. & 0.7160 \\
This work & A & 264 & 4,175 & \\
$10 \%$ FS & & & & 0.9415 \\
$100 \%$ FS & & & & 0.9580 \\
\hline
\end{tabular}

\begin{tabular}{|c|c|c|c|c|c|c|}
\hline \multicolumn{2}{|l|}{ Dataset } & \multicolumn{3}{|c|}{ Features } & \multicolumn{2}{|l|}{ F1 } \\
\hline Feature selection & Classification & $\mathrm{D}$ & $(\%)$ & $\mathrm{N}$ & KNN & SVM \\
\hline \multicolumn{7}{|l|}{ Audio } \\
\hline IOWA & ISMIR & 47 & $(7 \%)$ & 21 & 0.6891 & 0.7727 \\
\hline ISMIR & & 88 & $(13 \%)$ & 23 & 0.7171 & 0.7923 \\
\hline ISMIR & IOWA & 88 & $(13 \%)$ & 23 & 0.9182 & 0.9477 \\
\hline IOWA & & 47 & $(7 \%)$ & 21 & 0.9045 & 0.9449 \\
\hline \multicolumn{7}{|l|}{ Video } \\
\hline RAI-30 s & BBC $-30 \mathrm{~s}$ & 283 & $(42 \%)$ & 31 & 0.9670 & 0.9899 \\
\hline $\mathrm{BBC}-30 \mathrm{~s}$ & & 76 & $(11 \%)$ & 25 & 0.9606 & 0.9657 \\
\hline $\mathrm{BBC}-30 \mathrm{~s}$ & RAI-30 s & 76 & $(11 \%)$ & 25 & 0.9238 & 0.9184 \\
\hline RAI-30 s & & 283 & $(42 \%)$ & 31 & 0.8795 & 0.9415 \\
\hline
\end{tabular}

Feature selection is performed on $10 \%$ of the corresponding data with $t h_{c}=0.99$ well-defined feature set is competitive for a broad range of data and application settings. Therefore, in the following experiments we investigate the interrelation between data (and thus the underlying application scenario) and selected feature set, and its influence on the classification performance in more detail. Again, all experiments are based on 10 independent and randomly initialized runs and 10-fold cross-validated.

In our first experiment, we exchange the selected feature sets for the two audio datasets, ISMIR and IOWA, and for two video datasets, BBC and RAI (30 s segments), i.e., we employ features selected on the one dataset (e.g., ISMIR) to perform media classification on the second dataset related in terms of employed media (e.g., IOWA). Table 12 compares the achieved results in terms of F1-scores to the results using the original feature set, i.e., the features selected using the very same dataset as for the classification. The potentially expected drop in the classification scores cannot be confirmed. In fact, overall, the switch of the underlying feature sets leads to comparable performances for all datasets. The slight variations in the F1-scores indicate a dependency on the size of the feature set rather than on the dataset originally employed for the feature selection process. Therefore, we repeat the experiment with a comparable size of the selected feature set (in terms of resulting dimensionality). Additionally, we perform cross-media exchange of the selected feature sets to magnify potential dependencies between selected features and underlying data and application scenarios. Table 13 summarizes the achieved results. Again, the classification performance for a single dataset shows only slight deviations although the feature selection has been performed on partly strongly varying data and for a different application scenario. For example, the SVM classifier achieves an F1-score of $79.23 \%$ for the ISMIR audio data using features selected on the very same data in contrast to an F1-score of $78.76 \%$ using features selected on the dataset consisting of BBC documentary videos.

The results seem to support the assumption, that a welldefined feature set can be employed for a broad range of application scenarios. The question arises how to additionally increase the applicability of the selected feature set for varying data and application scenarios. We consider two possibilities. The first scenario accounts for the situation 
Table 13 Evaluation of the classification performance of feature selection on a different dataset

\begin{tabular}{|c|c|c|c|c|c|c|}
\hline \multicolumn{2}{|l|}{ Dataset } & \multicolumn{3}{|c|}{ Features } & \multicolumn{2}{|l|}{$\mathrm{F} 1$} \\
\hline Feature selection & Classification & $\mathrm{D}$ & $(\%)$ & $\mathrm{N}$ & $\mathrm{KNN}$ & SVM \\
\hline ISMIR (10\%) & ISMIR & 88 & $(13 \%)$ & 23 & 0.7171 & 0.7923 \\
\hline IOWA (20\%) & & 97 & $(14 \%)$ & 24 & 0.6723 & 0.7743 \\
\hline BBC-30 s (10\%) & & 76 & $(11 \%)$ & 25 & 0.6898 & 0.7876 \\
\hline RAI-30 s (1\%) & & 80 & $(12 \%)$ & 23 & 0.6998 & 0.7728 \\
\hline ISMIR (10\%) & IOWA & 88 & $(13 \%)$ & 23 & 0.9182 & 0.9477 \\
\hline IOWA (20\%) & & 97 & $(14 \%)$ & 24 & 0.8971 & 0.9506 \\
\hline BBC-30 s (10\%) & & 76 & $(11 \%)$ & 25 & 0.9036 & 0.9489 \\
\hline RAI-30 s (1\%) & & 80 & $(12 \%)$ & 23 & 0.9088 & 0.9430 \\
\hline ISMIR (10\%) & BBC-30 s & 88 & $(13 \%)$ & 23 & 0.9807 & 0.9647 \\
\hline IOWA (20\%) & & 97 & $(14 \%)$ & 24 & 0.9743 & 0.9755 \\
\hline BBC-30 s (10\%) & & 76 & $(11 \%)$ & 25 & 0.9606 & 0.9657 \\
\hline RAI-30 s (1\%) & & 80 & $(12 \%)$ & 23 & 0.9477 & 0.9622 \\
\hline ISMIR (10\%) & RAI-30 s & 88 & $(13 \%)$ & 23 & 0.9492 & 0.9192 \\
\hline IOWA (20\%) & & 97 & $(14 \%)$ & 24 & 0.9143 & 0.9202 \\
\hline BBC-30 s (10\%) & & 76 & $(11 \%)$ & 25 & 0.9238 & 0.9184 \\
\hline RAI-30 s (1\%) & & 80 & $(12 \%)$ & 23 & 0.9276 & 0.9090 \\
\hline
\end{tabular}

Feature selection is performed with $t h_{c}=0.99$, numbers in brackets denote the portion of data for feature selection where only a single dataset is available. Our previous experiment demonstrated that the analysis of a small portion of the data already allows for the selection of a competitive feature set (see Sect. 5.2). This is an especially efficient approach when working with very large datasets. However, there is no guarantee that even a random selection will cover most data characteristics of the underlying set. Increasing the selected data size for feature selection can increase the probability for a high portion of the present data characteristics (cp. Sect. 5.2). Nevertheless, the determination of the data size for feature selection is not a trivial task since it is strongly dependent on both the available data and on the specific data characteristics. Another approach to cover for broader data characteristics than in a single data portion is repeating the feature selection process and unifying the selected features. For this purpose in each run (out of ten), we perform feature selection for randomly selected data portions and merge the extracted features into an unified feature set. In order to provide for comparability of the results, we keep the corresponding data portion sizes as in our previous experiment: IOWA $20 \%$, ISMIR $10 \%$, BBC 10\%, and RAI 1\% (cp. Table 13). The second scenario accounts for the situation where multiple datasets are available. In this case, we merge the unified feature sets for all datasets into a single unified feature set (all data). Table 14 summarizes the achieved results and compares them to the performance of the original feature set (the result of a single data portion) and to the performance of the full feature set. Overall, the consideration of different portions of the data, unified feature set, improves the expressiveness of the selected features and leads to an increase in the classification performance in terms of F1-score in comparison to the original feature set. The employment of further datasets, unified feature set (all data), additionally improves the expressiveness of the selected feature set. Noteworthy is the fact that although this feature set has been selected in an unsupervised manner, it facilitates partly strongly differing data and application scenarios. Furthermore, the unified feature set (all data) outperforms previously achieved results (cp. Table 13) and it approaches the classification performance of the full feature set with significantly lower dimensionality $(31 \%) .^{5}$

The performed experiments confirm the fact that, in general, the employment of more features ${ }^{6}$ will most probably increase the classification performance of a feature set since different features usually capture different data characteristics. Hence, the results achieved by the full feature set outperform the results of the selected feature sets for all considered datasets. However, the addition of any further feature inevitably leads to an increase in both the dimensionality of the resulting feature set and the computational costs while the overall performance might only improve

\footnotetext{
5 The final unified feature set (all data) consists of the following 35 features (in alphabetical order): BTHI, CRMA, E4Hz, HMDV, HZZCR, LPZC, M7_AFF, M7_AH, M7_AP, M7_ASC, M7_ASF, M7_ASP, M7_ASS, M7_AW, M7_HSC, M7_HSD, M7_HSS, M7_HSV, M7_LAT, M7_SC, PTCH, PTCT, PTVB, ROFF, RPLP, RYPT, R_ZC, SF, SPCR, SPCT, SPDI, SPPS, STE, VDR, ZCR.

${ }^{6}$ Please note, that we do not consider noise as a feature.
} 
Table 14 Evaluation of the classification performance of the unified feature selection process for the different datasets

\begin{tabular}{|c|c|c|c|c|c|c|}
\hline \multirow[t]{2}{*}{ Dataset } & \multirow[t]{2}{*}{ Feature set } & \multicolumn{3}{|c|}{ Features } & \multicolumn{2}{|l|}{$\mathrm{F} 1$} \\
\hline & & $\mathrm{D}$ & $(\%)$ & $\mathrm{N}$ & $\mathrm{KNN}$ & SVM \\
\hline \multirow[t]{4}{*}{ ISMIR } & Original feature set & 88 & $(13 \%)$ & 23 & 0.7171 & 0.7923 \\
\hline & Unified feature set & 149 & $(22 \%)$ & 31 & 0.7470 & 0.8170 \\
\hline & Unified feature set (all data) & 213 & $(31 \%)$ & 35 & 0.7360 & 0.8340 \\
\hline & Full feature set & 679 & $(100 \%)$ & 50 & 0.7720 & 0.8550 \\
\hline \multirow[t]{4}{*}{ IOWA } & Original feature set & 97 & $(14 \%)$ & 24 & 0.8971 & 0.9506 \\
\hline & Unified feature set & 167 & $(25 \%)$ & 30 & 0.9180 & 0.9600 \\
\hline & Unified feature set (all data) & 213 & $(31 \%)$ & 35 & 0.9260 & 0.9660 \\
\hline & Full feature set & 679 & $(100 \%)$ & 50 & 0.9440 & 0.9780 \\
\hline \multirow[t]{4}{*}{$\mathrm{BBC}-30 \mathrm{~s}$} & Original feature set & 76 & $(11 \%)$ & 25 & 0.9606 & 0.9657 \\
\hline & Unified feature set & 115 & $(17 \%)$ & 31 & 0.9790 & 0.9840 \\
\hline & Unified feature set (all data) & 213 & $(31 \%)$ & 35 & 0.9890 & 0.9930 \\
\hline & Full feature set & 679 & $(100 \%)$ & 50 & 0.9910 & 0.9940 \\
\hline \multirow[t]{4}{*}{ RAI-30 s } & Original feature set & 80 & $(12 \%)$ & 23 & 0.9276 & 0.9090 \\
\hline & Unified feature set & 109 & $(16 \%)$ & 29 & 0.9460 & 0.9240 \\
\hline & Unified feature set (all data) & 213 & $(31 \%)$ & 35 & 0.9500 & 0.9480 \\
\hline & Full feature set & 679 & $(100 \%)$ & 50 & 0.9790 & 0.9800 \\
\hline
\end{tabular}

Feature selection is performed with $t h_{c}=0.99$ marginally. Therefore, feature selection is commonly applied to reduce dimensionality and computational costs. The main drawback of existing approaches is that they are tailored to specific data and/or application scenario. Any alteration of the data settings or the underlying application scenario requires a reconsideration of the employed features. In contrast, our last experiments demonstrate that the proposed unsupervised feature selection approach allows for the identification of a highly expressive feature set that can be applied for different data and application scenarios even if the feature selection is performed on a small portion of the available data. This is a crucial benefit in a dynamic environment where data continuously changes and application scenarios are subject to adaptation.

\section{Conclusion}

In this paper, we presented an unsupervised approach for the selection of robust multi-dimensional (group) features that exploits canonical correlation in order to separate relevant from less relevant features. In contrast to related works in the context of audio and video classification, the proposed approach preserves the original grouping of feature components into multi-dimensional (group) features, which results in a semantically interpretable feature selection. The approach is generic as it does not make any assumptions about the underlying data (or even application or task) characteristics, but it autonomously selects a feature set that efficiently describes the data. In addition, the feature set selected by our approach is remarkably robust: different runs or even experiments for different tasks show little variations in the set of selected features, even if the underlying training set varies considerably. We performed experiments on various audio and video datasets representing different application scenarios and data characteristics such as a strongly varying number of samples and classes and a varying class distribution. Achieved results show that our unsupervised approach is competitive to related works which were designed to solve very specific tasks and additionally demonstrates extraordinary robustness.

The reported experiments investigating the interrelation between selected features and provided data reveal some valuable insights. Achieved results indicate that the dependencies between data (and application scenario), and selected features are weaker than actually expected. The same wellselected features can discriminate both between viola and violin sounds and between different types of video documentaries, for example. An approach which is highly tailored to a particular task will most probably additionally improve the performance in terms of classification accuracy and/or feature dimensionality. However, any alteration of the task (such as the consideration of an additional label) will require the reconsideration of the employed features. In contrast, the performed experiments indicate that a single, highly expressive feature set can be applied for different data and application scenarios achieving highly competitive results. Such a robust data-driven approach is the only possibility when mining dynamic or unknown data collections where no prior information about the data is available. We believe that more 
attention and research time should be paid in the future to the general question of how to obtain more robust features in such environments.

Acknowledgements Open access funding provided by TU Wien (TUW). This work has been partly funded by the Vienna Science and Technology Fund (WWTF) through project ICT12-010. The authors would like to thank Maurizio Montagnuolo from RAI Centre for Research and Technological Innovation for providing the RAI TV dataset.

Open Access This article is distributed under the terms of the Creative Commons Attribution 4.0 International License (http://creativecomm ons.org/licenses/by/4.0/), which permits unrestricted use, distribution, and reproduction in any medium, provided you give appropriate credit to the original author(s) and the source, provide a link to the Creative Commons license, and indicate if changes were made.

\section{References}

1. Babu MM (2004) Introduction to microarray data analysis. Comput Genom Theory Appl 17(6):225-249

2. Baniya BK, Ghimire D, Lee J (2015) Automatic music genre classification using timbral texture and rhythmic content features. In: International conference on advanced communication technology, pp 434-443

3. Benetos E, Kotti M, Kotropoulos C (2006) Musical instrument classification using non-negative matrix factorization algorithms and subset feature selection. In: IEEE international conference on acoustics, speech and signal processing, vol 5, pp 1844-1847

4. Berrar DP, Dubitzky W, Granzow M et al (2003) A practical approach to microarray data analysis. Springer, New York

5. Breiman L (2001) Random forests. Mach Learn 45(1):5-32

6. Cover T, Hart P (1967) Nearest neighbor pattern classification. IEEE Trans Inf Theory 13(1):21-27

7. Dash M, Liu H (1997) Feature selection for classification. Intell Data Anal 1:131-156

8. Deng J, Simmermacher C, Cranefield S (2008) A study on feature analysis for musical instrument classification. IEEE Trans Syst Man Cybern B Cybern 38(2):429-438

9. Doraisamy S, Golzari S, Norowi NM, Sulaiman MN, Udzir NI (2008) A study on feature selection and classification techniques for automatic genre classification of traditional malay music. In: International conference on music information retrieval, pp 331336

10. Duda RO, Hart PE, Stork DG (2000) Pattern classification. WileyInterscience, New York, NY

11. Ekenel HK, Semela T (2013) Multimodal genre classification of TV programs and YouTube videos. Multimed Tools Appl 63(2):547567

12. Fiebrink R, Fujinaga I (2006) Feature selection pitfalls and music classification. In: International conference on music information retrieval

13. Friedman J, Hastie T, Tibshirani R (2010) A note on the group lasso and a sparse group lasso. ArXiv e-prints

14. Fu Z, Lu G, Ting KM, Zhang D (2011) A survey of audiobased music classification and annotation. IEEE Trans Multimedia 13(2):303-319

15. Guyon I, Elisseeff A (2003) An introduction to variable and feature selection. J Mach Learn Res 3:1157-1182

16. Hall MA (1999) Correlation-based feature subset selection for machine learning. Ph.D. thesis, University of Waikato
17. Hall MA, Holmes G (2003) Benchmarking attribute selection techniques for discrete class data mining. IEEE Trans Knowl Data Eng 15(6): 1437-1447

18. Hall P, Miller H (2009) Using generalized correlation to effect variable selection in very high dimensional problems. J Comput Graph Stat 18(3):533-550

19. Hossain MZ, Kabir MM, Shahjahan M (2016) A robust feature selection system with Colin's CCA network. Neurocomputing $173: 855-863$

20. Hotelling $H$ (1936) Relations between two sets of variates. Biometrika 28(3/4):321-377

21. Huang GB, Zhu QY, Siew CK (2006) Extreme learning machine: theory and applications. Neurocomputing 70(1-3):489-501

22. Huang YF, Lin SM, Wu HY, Li YS (2014) Editorial: music genre classification based on local feature selection using a self-adaptive harmony search algorithm. Data Knowl Eng 92:60-76

23. IOWA Dataset: Univ. of Iowa musical instrument samples. http:// theremin.music.uiowa.edu (1997)

24. ISMIR 2004 Dataset: International conference on music information retrieval—audio description contest data set. http://ismir2004. ismir.net/ (2004)

25. Jang D, Jang SJ (2014) Very short feature vector for music genre classification based on distance metric learning. In: International conference on audio, language and image processing, pp 726-729

26. Jang D, Jin M, Yoo CD (2008) Music genre classification using novel features and a weighted voting method. In: IEEE international conference on multimedia and expo, pp 1377-1380

27. Kim S, Georgiou P, Narayanan S (2013) On-line genre classification of TV programs using audio content. In: IEEE international conference on acoustics, speech and signal processing, pp 798-802

28. Kohavi R, John GH (1997) Wrappers for feature subset selection. Artif Intell 97(1-2):273-324

29. Kononenko I (1994) Estimating attributes: analysis and extensions of RELIEF. In: European conference on machine learning, pp 171182

30. Lee CH, Shih JL, Yu KM, Lin HS (2009) Automatic music genre classification based on modulation spectral analysis of spectral and cepstral features. IEEE Trans Multimedia 11(4):670-682

31. Levandowsky M, Winter D (1971) Distance between sets. Nature 234(5323):34-35

32. Lim SC, Lee JS, Jang SJ, Lee SP, Kim MY (2012) Music-genre classification system based on spectro-temporal features and feature selection. IEEE Trans Consum Electron 58(4):1262-1268

33. Liu H, Setiono R (1995) Chi2: feature selection and discretization of numeric attributes. In: International conference on tools with artificial intelligence, pp 388-391

34. Liu H, Setiono R (1996) A probabilistic approach to feature selection-a filter solution. In: International conference on machine learning, pp 319-327

35. Meier L, Geer SVD, Bühlmann P (2008) The group lasso for logistic regression. J R Stat Soc Series B Stat Methodol 70(1):53-71

36. Mitrovic D, Zeppelzauer M, Breiteneder C (2010) Features for content-based audio retrieval. In: Advances in computers: improving the web, vol 78, chap 3, pp 71-150. Elsevier

37. Montagnuolo M, Messina A (2007) TV genre classification using multimodal information and multilayer perceptrons. In: AI*IA 2007: artificial intelligence and human-oriented computing, pp 730-741

38. Montagnuolo M, Messina A (2009) Parallel neural networks for multimodal video genre classification. Multimed Tools Appl 41(1):125-159

39. Nie F, Huang H, Cai X, Ding CH (2010) Efficient and robust feature selection via joint $\ell 2,1$-norms minimization. In: International conference on neural information processing systems, pp 1813-1821

40. Nielsen AB, Sigurdsson S, Hansen LK, Arenas-Garcia J (2007) On the relevance of spectral features for instrument classification. 
In: IEEE international conference on acoustics, speech and signal processing, vol 2, pp 485-488

41. Pascoal C, De Oliveira MR, Valadas R, Filzmoser P, Salvador P, Pacheco A (2012) Robust feature selection and robust PCA for internet traffic anomaly detection. In: IEEE international conference on computer communications (INFOCOM), pp 1755-1763

42. Quinlan JR (1993) C4.5: programs for machine learning. Morgan Kaufmann Publishers Inc., San Francisco, CA, USA

43. Saari P, Eerola T, Lartillot O (2011) Generalizability and simplicity as criteria in feature selection: application to mood classification in music. IEEE Trans Audio Speech Lang Process 19(6):1802-1812

44. Saeys Y, Inza I, Larrañaga P (2007) A review of feature selection techniques in bioinformatics. Bioinformatics 23(19):2507-2517

45. Sageder G, Zaharieva M, Breiteneder C (2016) Group feature selection for audio-based video genre classification. In: International conference on multimedia modeling, pp 29-41

46. Sageder G, Zaharieva M, Zeppelzauer M (2014) Unsupervised selection of robust audio feature subsets. In: SIAM international conference on data mining, pp 686-694

47. Sargent G, Hanna P, Nicolas H, Bimbot F (2015) Exploring the complementarity of audio-visual structural regularities for the classification of videos into tv-program collections. In: IEEE international symposium on multimedia (ISM), pp 620-623

48. Seo JS, Lee S (2011) Higher-order moments for musical genre classification. Signal Process 91(8):2154-2157

49. Seyerlehner K, Schedl M, Pohle T, Knees P (2010) Using blocklevel features for genre classification, tag classification and music similarity estimation. In: Music information retrieval evaluation exchange

50. Silla C, Koerich A, Kaestner C (2008) Feature selection in automatic music genre classification. In: IEEE international symposium on multimedia, pp 39-44
51. Simmermacher C, Deng D, Cranefield S (2006) Feature analysis and classification of classical musical instruments: an empirical study. In: Industrial conference on data mining: advances in data mining. Applications in medicine, web mining, marketing, image and signal mining, pp 444-458

52. Simon N, Friedman J, Hastie T, Tibshirani R (2013) A sparse-group lasso. J Comput Graph Stat 22(2):231-245

53. Somol P, Novovicova J (2010) Evaluating stability and comparing output of feature selectors that optimize feature subset cardinality. IEEE Trans Pattern Anal Mach Intell 32(11):1921-1939

54. Song Q, Ni J, Wang G (2013) A fast clustering-based feature subset selection algorithm for high-dimensional data. IEEE Trans Knowl Data Eng 25(1):1-14

55. Tibshirani R (1997) The lasso method for variable selection in the cox model. In: Statistics in medicine, pp 385-395

56. Vapnik V (1995) The nature of statistical learning theory. Springer

57. Xu Z, Huang G, Weinberger KQ, Zheng AX (2014) Gradient boosted feature selection. In: ACM SIGKDD international conference on knowledge discovery and data mining, pp 522-531

58. Yang F, Mao K (2011) Robust feature selection for microarray data based on multicriterion fusion. IEEE/ACM Trans Comput Biol Bioinform (TCBB) 8(4):1080-1092

59. Yang YH, Liu CC, Chen HH (2006) Music emotion classification: a fuzzy approach. In: ACM international conference on multimedia, pp 81-84

60. Yaslan Y, Cataltepe Z (2006) Audio music genre classification using different classifiers and feature selection methods. In: International conference on pattern recognition, pp 573-576

61. Yu L, Liu H (2004) Efficient feature selection via analysis of relevance and redundancy. J Mach Learn Res 5:1205-1224

62. Yuan M, Lin Y (2006) Model selection and estimation in regression with grouped variables. J R Stat Soc Series B 68:49-67 